\title{
Novel Miniaturized Equal/Unequal HMSIW Filtering Power Dividers Based on the Evanescent Mode Technique
}

\author{
Mostafa DANAEIAN \\ Department of Electrical Engineering, Vali-e-Asr University of Rafsanjan, Rafsanjan, Iran \\ danaeian@vru.ac.ir
}

Submitted June 6, 2020 / Accepted November 11, 2020

\begin{abstract}
In this paper, three miniaturized equal/unequal filtering power dividers (FPDs) applying the half-mode substrate integrated waveguide (HMSIW) and metamaterial concepts are offered. The operational method of the presented structures is based on the theory of evanescent mode propagation. The stepped-impedance resonator (SIR) technique has been employed to reduce the dimension of the conventional complementary split ring resonator (CSRR) unit-cell. In this technique, the slot lines in the conventional CSRR are replaced by the stepped-impedance slot lines in the improved metamaterial unit-cell which is called SIR-CSRR unit-cell. By means of the SIR-CSRR unitcell, three equal/unequal FPDs with arbitrary power-dividing ratio have been reported. Additionally, to further size reduction of the proposed FPDs, the HMSIW platforms are used. All of the proposed HMSIW FPDs are designed at $2.4 \mathrm{GHz}$ which are suitable for WLAN applications. For demonstration of the applied procedures in the proposed HMSIW FPDs, the suggested equal/unequal HMSIW FPDs have been fabricated and measured. A reasonable agreement between simulated and measured results has been achieved. The entire size of the suggested equal/unequal HMSIW FPDs is about $0.11 \times 0.09 \lambda_{g}^{2}$.
\end{abstract}

\section{Keywords}

Filtering power divider (FPD), half mode substrate integrated waveguide (HMSIW), evanescent mode technique, electric dipoles, stepped-impedance resonators, arbitrary power division and miniaturization

\section{Introduction}

The bandpass filters (BPFs) and power dividers (PDs) are the fundamental passive components in the microwave systems [1-5]. Microwave engineers attempted to design and introduce many types of BPFs and PDs to attain more ideal specifications and performances. Integrating two or more single-function circuits into one block is an interest- ing procedure to achieve the size reduction, low cost, low loss and high performance. Filtering power divider (FPD) is a dual-functional component which is realized by combining the BPF and PD [6-14]. Numerous FPDs based on the several methods have been developed in [6-19]. The main drawbacks of the reported circuits can be mentioned the large size, high-insertion loss, high cost and difficult manufacturing.

The concept of substrate integrated waveguide (SIW) has been introduced to overcome the weaknesses of the conventional waveguide. The SIW platform is manufactured in a planar substrate and can be implemented with two metal plates on the top and bottom sides which are connected with linear arrays of the metallized via holes. The SIW structures have many benefits like low cost, low loss, high quality factor and easy integration with planar circuits however, occupies a large area. Therefore, this configuration needs a technique to decrease its dimension. Half-mode SIW (HMSIW) [20], folded SIW (FSIW) [21], ridge SIW (RSIW) [22] and the evanescent mode propagation technique [23] are some of methods to reduce the size of the conventional SIW structures.

In this paper, three miniaturized FPDs with an arbitrary power division ratio founded on the HMSIW configurations and by using metamaterial concept are introduced. To achieve the compact size and the bandpass filtering response, the evanescent mode theory has been employed. Based on this technique, an additional forward passband below the SIW cutoff frequency could be obtained by engraving the electric dipoles on the SIW surface. Furthermore, approximately $50 \%$ size reduction can be achieved by using the HMSIW technique without damaging the performance of the SIW structure. In the proposed FPDs, the arbitrary power division ratio can be straightforwardly obtained by changing the places of the output ports. In order to confirm the distinctive capabilities of the proposed structures, three HMSIW FPDs with different power division ratios of $1: 1,1: 4$, and 1:8 have been designed, simulated, fabricated and tested. The proposed FPDs have the advantages in terms of low cost, low loss, high selectivity, high power-handling capability and easy integration with planar circuits. 


\section{Design Procedure of the Proposed HMSIW PDs}

The stepped-impedance resonator (SIR) is the attractive technique to miniaturize the metamaterial unit-cells [1], [2]. Based on this technique, a metamaterial unit-cell has been reported which is called the SIR-CSRR [1], [2]. The SIR-CSRR unit-cell consists of two modified rings so that the conventional slot lines is replaced by the SIR slot lines [1], [2]. By replacing the SIR transmission lines in the metamaterial structure instead of the conventional transmission lines, a downward frequency shift in the S-parameters has been achieved. Consequently, the electrical size of the SIR-CSRR unit-cell is smaller than the conventional complementary split-ring resonator (CSRR) with the same size. It means that the physical size of the SIR-CSRR unitcell is decreased in comparison with the conventional CSRR unit-cell with the same central frequency.

Figure 1 depicts the traditional CSRR unit-cell and the SIR-CSRR unit-cell, respectively with the identical sizes. In the condition of the same sizes, the resonance frequency of the SIR-CSRR unit-cell is less than the conventional CSRR [1], [2]. Similarly to the conventional CSRR, the SIR-CSRR unit-cell is also modelled as a shunt resonant tank with inductance $L_{\mathrm{r}}$ and capacitance $C_{\mathrm{r}}$. The resonance frequency of the SIR-CSRR unit-cell is able to achieve from [1], [2]:

$$
f_{\mathrm{r}}=\frac{1}{2 \pi \sqrt{L_{\mathrm{r}} C_{\mathrm{r}}}} .
$$

The CSRR and SIR-CSRR unit-cells, which are excited by an axial external electric field, act as electric dipoles. In fact, these unit-cells are vital components to implement the $\varepsilon$-negative media in the neighborhood of their resonant frequencies. Consequently, a $\varepsilon$-negative can be predictable in a narrow band near their resonant frequencies.

On the other hand, the SIW structure is able to provide $\varepsilon$-negative when worked below the cutoff frequency of its dominant $\mathrm{TE}_{10}$ mode [24]. The dispersion constant of the SIW configuration can be calculated with the following relation [24]:

$$
k=\omega \sqrt{\mu_{\mathrm{r}} \varepsilon_{\mathrm{eff}}}, \quad \varepsilon_{\mathrm{eff}}=\varepsilon_{\mathrm{r}}\left(1-\frac{\omega_{0}^{2}}{\omega^{2}}\right)
$$

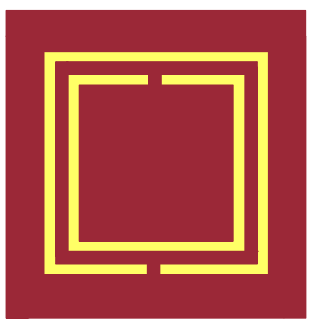

(a)

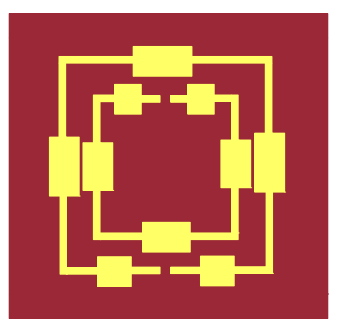

(b)
Fig. 1. Configuration of the (a) conventional CSRR unit-cell and (b) SIR-CSRR unit-cell. where $\mu_{\mathrm{r}}$ and $\varepsilon_{\mathrm{r}}$ are the permeability and permittivity of the substrate which the SIW structure is implemented on it, and $\omega_{0}$ is the cutoff frequency for the dominant $\mathrm{TE}_{10}$ mode. When the SIW is utilized below the cutoff frequency, $\varepsilon_{\text {eff }}$ becomes negative and $k$ becomes an imaginary number. In this case, the wave propagation is prohibited and the resulting mode is an evanescent mode. Accordingly, a uniform $\varepsilon$-negative material can be automatically achieved in the frequency range below the SIW cutoff frequency [1], [2]. This property looks to be useless for the typical SIW applications while it becomes an enchanting feature under the scenario of metamaterial concept. Consequently, a seductive technique for combining the metamaterial and SIW structures is presented which calls the evanescent mode propagation technique. Based on the theory of evanescent mode, by engraving the electric dipoles on the metal surface of the SIW structure, an additional passband below the SIW cutoff frequency can be achieved. This means that, by combining the SIW structure and a $\varepsilon$-negative material, the intrinsic constitutive parameters of the SIW structure below the cut-off frequency have been changed and a forward wave propagation can be obtained [1], [2]. Since the proposed SIR-CSRR act as a $\varepsilon$-negative material, therefore a forward passband below the cut-off frequency of the SIW structure is achieved by etching the SIR-CSRR unit-cell on the SIW structure. Furthermore, the HMSIW structure has the advantages of the SIW and its size is nearly half of the SIW which has been obtained with cutting plane along the magnetic wall. Therefore, by loading the proposed SIR-CSRR unit-cell on the metal surface of the HMSIW structure, the physical size of the filter has been reduced which means that miniaturization has been occurred. The configuration of the HMSIW filter loaded by the SIR-CSRR is depicted in Fig. 2. The simulated frequency responses for this filter are illustrated in Fig. 3. As shown in this figure, a passband appears in the vicinity of $f_{\mathrm{r}}$ $=2.4 \mathrm{GHz}$ below the cutoff frequency of the HMSIW structure.

Extracted real part of the permittivity $(\varepsilon)$ and permeability $(\mu)$ of the designed filter is displayed in Fig. 4. As can be seen in this figure, the positive permittivity is occurred at $2.4 \mathrm{GHz}$ which is in the neighborhood of the resonance frequency of the SIR-CSRR unit-cell. This validates that by loading the SIR-CSRR unit-cell on the HMSIW configuration, original $\varepsilon$-negative material below the HMSIW cutoff frequency becomes $\varepsilon$-positive and a forward propagation has been achieved. In other words, the SIR-CSRR unit-cell is able to convert the negative permittivity of the intrinsic material into positive in a limited frequency range. The normalized dispersion relation and the curve of the attenuation constant of the HMSIW filter loaded by the SIR-CSRR unit-cell are also depicted in Fig. 5. As illustrated in this figure, the dispersion diagram shows a positive slope at that frequencies which the SIRCSRR unit-cell is excited by an axial electric field. This curve confirms that the passband which has been occurring below the HMSIW cutoff frequency has forward-wave nature. As well as, the curve of the attenuation constant 
demonstrates that there are two passbands which one of them is the forward-wave passband below the cutoff frequency of the HMSIW platform and another passband is the inherent HMSIW high passband. The simulated unwrapped phase of the designed filter is shown in Fig. 6. As proved in Fig. 6, the phase is negative in the frequency region which the bandpass is occurring and confirms that this bandpass is because of the forward wave propagation below the HMSIW cutoff frequency.

In order to investigate the design procedure, the equivalent circuit model of the proposed HMSIW filter loaded by the SIR-CSRR unit-cell is illustrated in Fig. 7(a). In this model, the HMSIW can be considered as an ordinary two-wire transmission line loaded with infinite number of via holes which are modeled as inductance $L_{\text {via }}$. This component makes a high-pass responses of the HMSIW structure. The proposed SIR-CSRR unit-cell can be modeled as a resonator tank with inductance $L_{\mathrm{r}}$ and capacitance $C_{\mathrm{r}}$. As well as, the coupling between the HMSIW structure and the SIR-CSRR unit-cell has been considered as a combination of electric and magnetic couplings. Accordingly, $L_{\mathrm{c}}$ and $C_{\mathrm{c}}$ are used to illustrate the inductive and capacitive couplings between the HMSIW structure and the SIRCSRR unit-cell. The resulting element values of the circuit model for the designed HMSIW filter loaded by the SIRCSRR unit-cell are: $L_{\text {via }}=4.4 \mathrm{nH}, C_{\mathrm{r}}=2.65 \mathrm{pF}, L_{\mathrm{r}}=$ $2.3 \mathrm{nH}, C_{\mathrm{c}}=2.1 \mathrm{pF}, L_{\mathrm{c}}=0.98 \mathrm{nH}$. These lumped element values have been calculated using the Advance Design System (ADS) electromagnetic simulator.

The simulated frequency responses of the proposed HMSIW band pass filter loaded by the SIR-CSRR unit-cell and its equivalent circuit model are shown in Fig. 7(b). As well as, the simulated frequency responses of the conventional HMSIW are illustrated in Fig. 7(b). As can be seen in this figure, the transmission pole and zero in the frequency responses for the proposed filter and its equivalent circuit model are closely matched with each other. It should be noted that the circuit model cannot reflect the influence of the high-order modes of the designed filter because it is simplified and valid only for a limited frequency range. The circuit model gives a zero-transmission frequency at:

$$
f_{\mathrm{z}}=\frac{1}{2 \pi \sqrt{L_{\mathrm{c}} C_{\mathrm{c}}}} .
$$

It is worth mentioning that the series part impedance in the circuit model undergoes an abrupt change (from inductance infinity to capacitance infinity) around $3.4 \mathrm{GHz}$ which is a sudden change on the dispersion diagram shown in Fig. 5.

On the other hands, according to the equivalent circuit model, the influence of the wider slot line on the SIRCSRR configuration can be considered as an increase in $L_{\mathrm{r}}$ in the circuit model. Consequently, the resonance frequency becomes lower compared to the conventional CSRR. In other words, by applying the SIR technique in the conventional CSRR unit-cells, the resonance frequency of the proposed SIR-CSRR unit-cells will be shifted down because of increasing the total inductance of them.

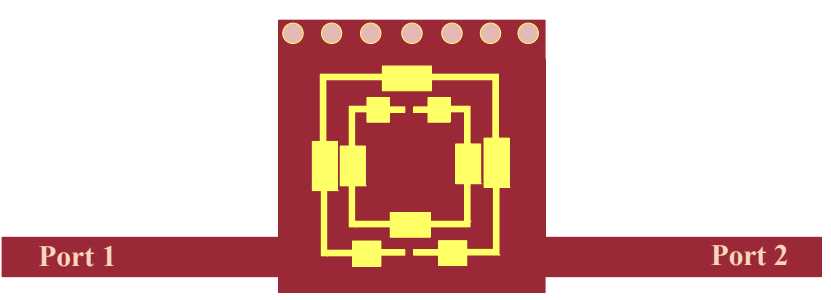

Fig. 2. Configuration of the HMSIW bandpass filter loaded by the SIR-CSRR unit-cell.

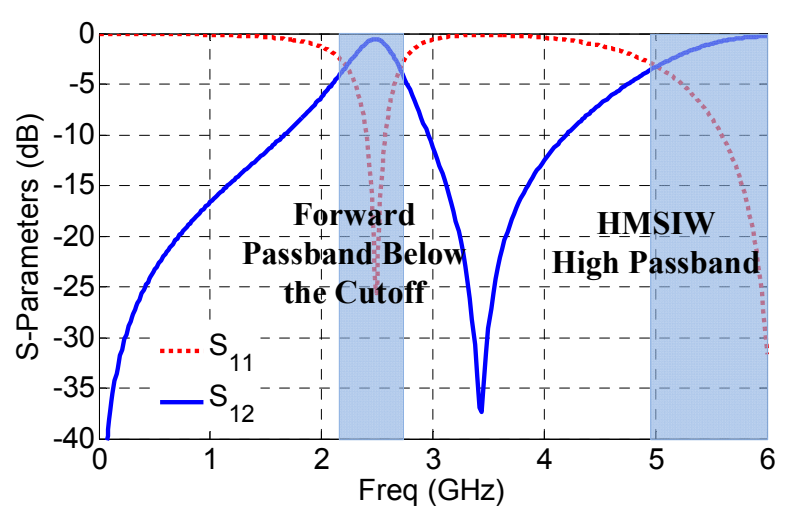

Fig. 3. Simulated frequency responses of the HMSIW bandpass filter loaded by the SIR-CSRR unit-cell.

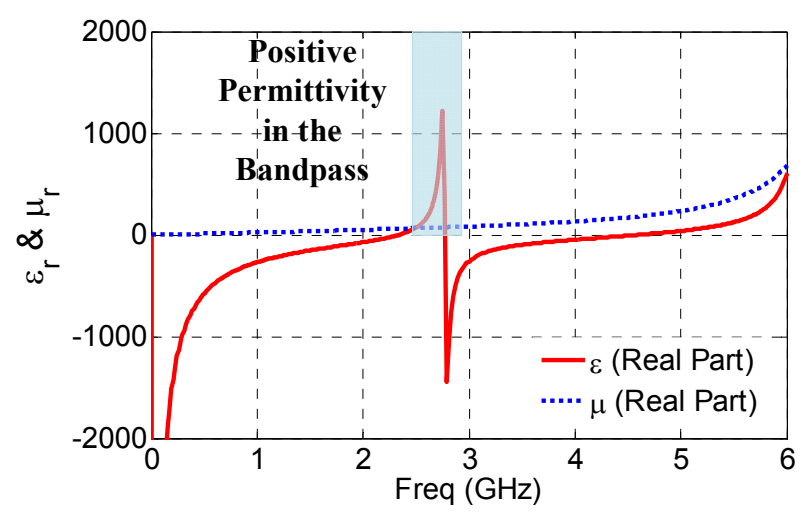

Fig. 4. Extracted real part of the relative permittivity $\left(\varepsilon_{\mathrm{r}}\right)$ and relative permeability $\left(\mu_{\mathrm{r}}\right)$ for the HMSIW bandpass filter loaded by the SIR-CSRR unit-cell.

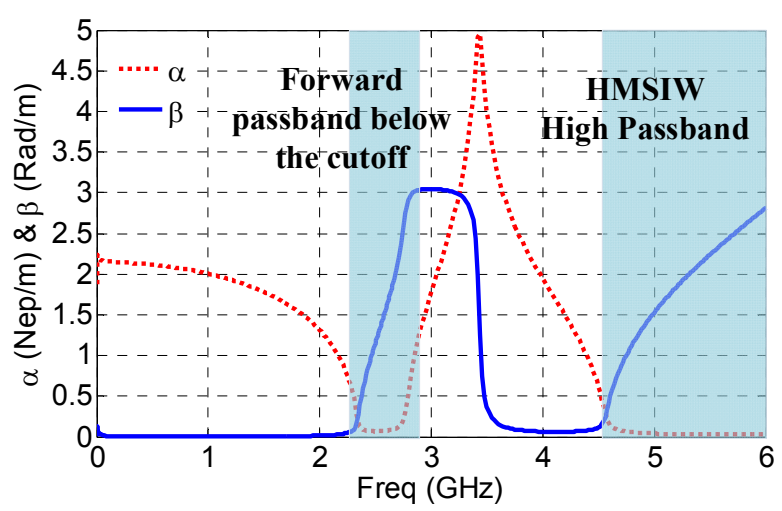

Fig. 5. Dispersion and attenuation diagrams for the HMSIW bandpass filter loaded by the SIR-CSRR unit-cell. 


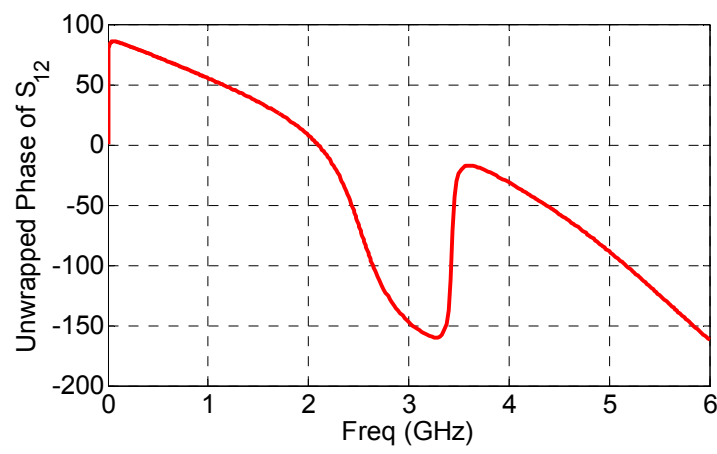

Fig. 6. Simulated unwrapped phase for the HMSIW bandpass filter loaded by the SIR-CSRR unit-cell.

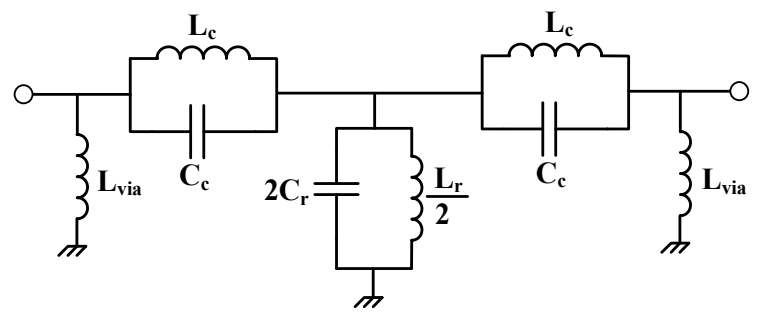

(a)

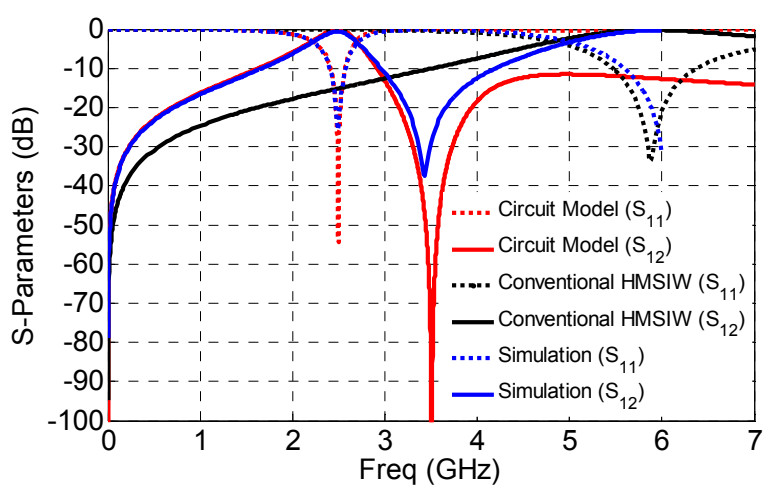

(b)

Fig. 7. (a) Equivalent-circuit model and (b) frequency responses of the conventional HMSIW, circuit model and EM-simulated of the HMSIW filter loaded by the proposed SIR-CSRR unit-cell.

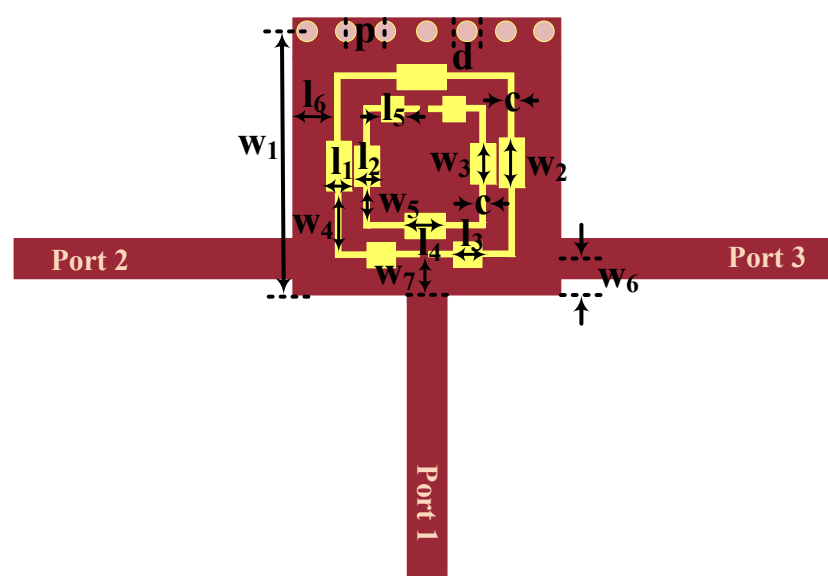

Fig. 8. Configuration of the proposed equal HMSIW FPD.

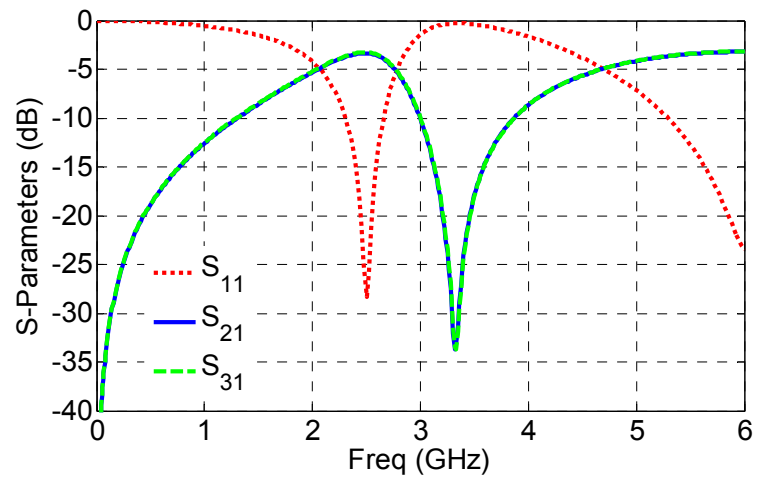

Fig. 9. Simulated results of the proposed equal HMSIW FPD.

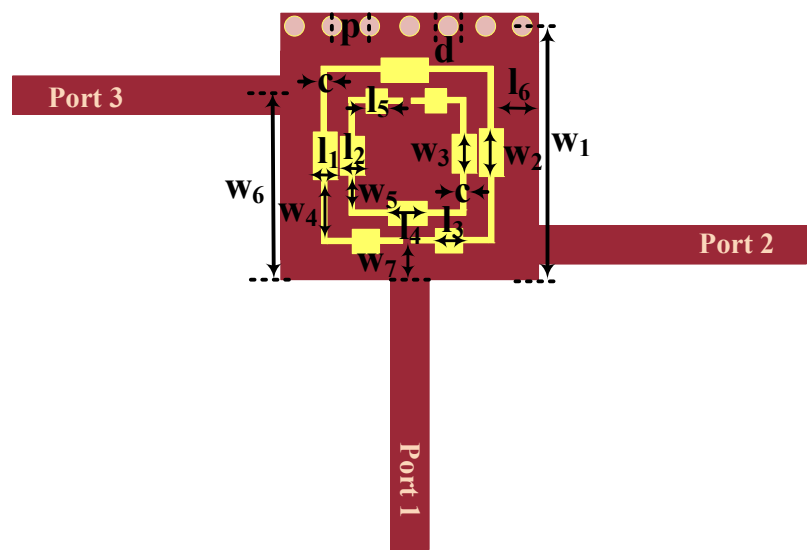

Fig. 10. Configuration of the proposed unequal HMSIW FPD.

Figure 8 illustrates the suggested equal FPD using HMSIW structure. The simulated frequency responses of the proposed equal HMSIW FPD are depicted in Fig. 9. To attain an ultra-compact size, the HMSIW configuration has been used. As previously mentioned, the working principle of the designed FPDs is based on the evanescent mode technique. According to this technique, an extra forward passband below the cutoff frequency of the HMSIW structure can be achieved. The filtering property of the suggested FPDs is because of the using this technique. In all of the proposed FPDs, the center frequency of the passband can be simply tuned by resizing the SIR-CSRR unitcell. As well as, the bandwidth and matching performance can be adjusted by changing the values of $l_{6}$ and $w_{7}$. Additionally, the power division ratios of the proposed FPDs can be regulated and controlled by varying the value of $w_{6}$.

The suggested unequal FPD is shown in Fig. 10. Figure 11 shows the simulated frequency responses of this layout. As demonstrated in these figures, different power division ratios have been achieved by altering the locations of the output ports which two FPDs with power division ratios of $1: 4$ and $1: 8$ are realized by this structure, respectively. The curve of the changes in the power division ratio with altering the value of $w_{6}$ (distance between the two output ports) is plotted in Fig. 12. The power division ratio can be increased by raising the value of $w_{6}$. 


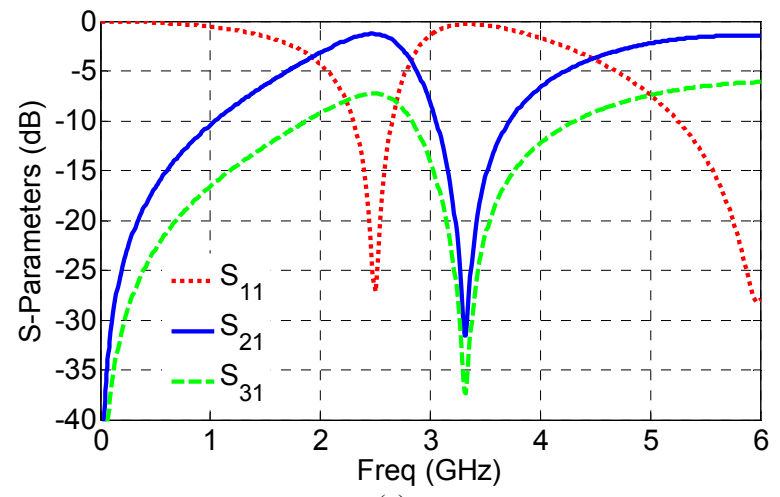

(a)

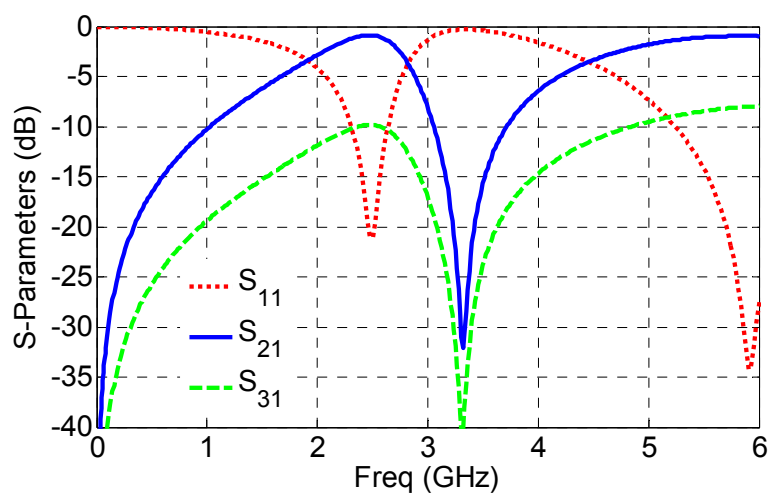

(b)

Fig. 11. Simulated results of the unequal HMSIW FPD: (a) $\Delta$ out $=6 \mathrm{~dB}$ and (b) $\Delta$ out $=9 \mathrm{~dB}$.

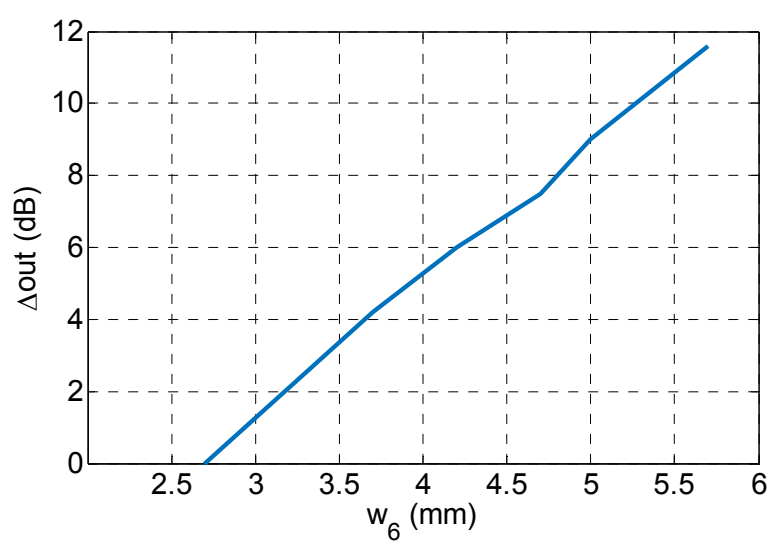

Fig. 12. The $\triangle$ out of the proposed FPDs as a function of $\mathrm{w}_{6}$.

The design steps and optimization processes of the dimensions for the proposed FPDs have been summarized as follows:

1. The cutoff frequency of the dominant $\mathrm{TE}_{10}$ mode for the proposed FPDs can be tuned by altering the values of $\mathrm{w}_{1}, \mathrm{~d}$, and $\mathrm{p}$.

2. The center frequency of the proposed FPDs can be adjusted by the values of $\mathrm{w}_{2}-\mathrm{w}_{5}, 1_{1}-1_{5}$ and $\mathrm{c}$.

3. The power division ratios of the proposed FPDs can be controlled by changing the distance $\mathrm{w}_{6}$.

4. The bandwidth and matching performance of the proposed FPDs can be regulated by varying the values of $1_{6}$ and $\mathrm{w}_{7}$.

\section{Results and Discussion}

The whole suggested HMSIW FPDs are implemented on the Rogers RO4003C substrate with a dielectric constant of 3.55, loss tangent of 0.0027 and thickness of $0.508 \mathrm{~mm}$. The final sizes and parameters of the proposed equal/unequal FPDs are: $\mathrm{w}_{1}=7.5 \mathrm{~mm}, \mathrm{w}_{2}=1.6 \mathrm{~mm}$, $\mathrm{w}_{3}=1.1 \mathrm{~mm}, \mathrm{w}_{4}=1.8 \mathrm{~mm}, \mathrm{w}_{5}=1.1 \mathrm{~mm}, \mathrm{l}_{1}=0.8 \mathrm{~mm}$, $1_{2}=0.8 \mathrm{~mm}, 1_{3}=0.8 \mathrm{~mm}, l_{4}=1.2 \mathrm{~mm}, 1_{5}=0.6 \mathrm{~mm}$, $\mathrm{c}=0.2 \mathrm{~mm}, \mathrm{~d}=0.8 \mathrm{~mm}, \mathrm{p}=1.4 \mathrm{~mm}, \mathrm{w}_{6}(\Delta$ out $=0 \mathrm{~dB})=$ $2.7 \mathrm{~mm}, \mathrm{w}_{6}(\Delta$ out $=6 \mathrm{~dB})=4.2 \mathrm{~mm}, \mathrm{w}_{6}(\Delta$ out $=9 \mathrm{~dB})=$ $5 \mathrm{~mm}, \mathrm{l}_{6}=0.4 \mathrm{~mm}$, and $\mathrm{w}_{7}=1.5 \mathrm{~mm}$. The overall dimension of the proposed equal/unequal FPDs is $0.11 \times 0.09 \lambda_{\mathrm{g}}{ }^{2}$. The photographs of the fabricated equal/unequal FPDs are shown in Fig. 13.

Comparison between the simulated and measured results for the equal/unequal HMSIW FPDs are shown in Fig. 14 and Fig. 15.

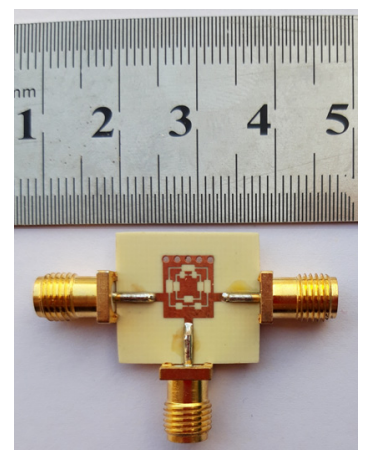

(a)

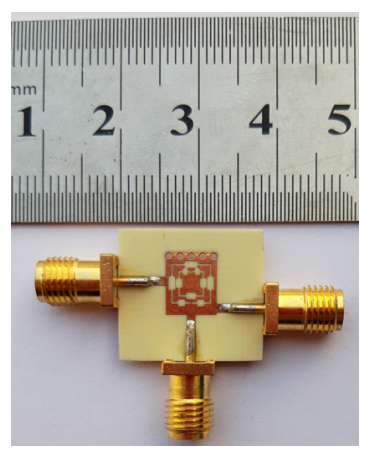

(b)

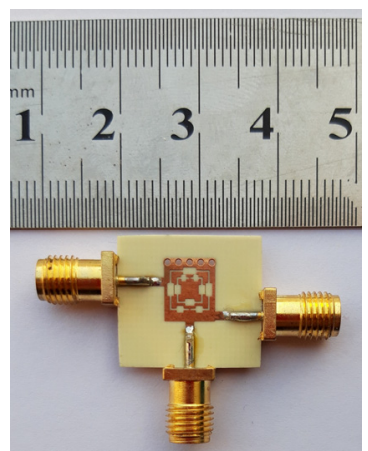

(c)

Fig. 13. Photograph of the fabricated equal/unequal HMSIW FPDs: (a) $\Delta$ out $=0 \mathrm{~dB}, \quad$ (b) $\Delta$ out $=6 \mathrm{~dB}$ and (c) $\Delta$ out $=9 \mathrm{~dB}$. 


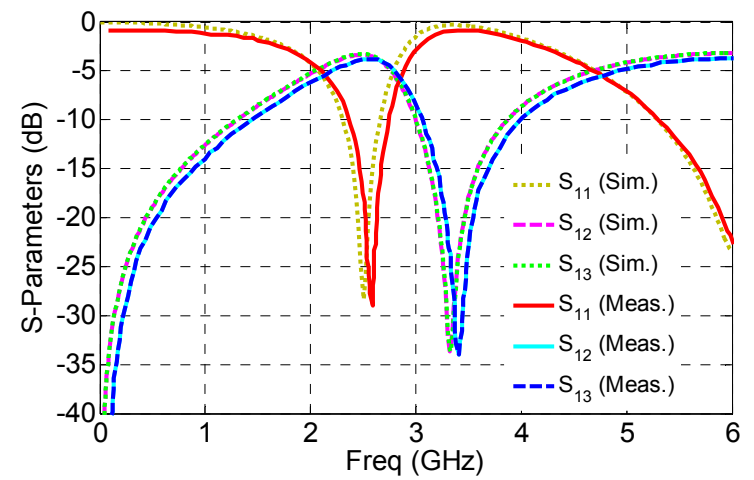

(a)

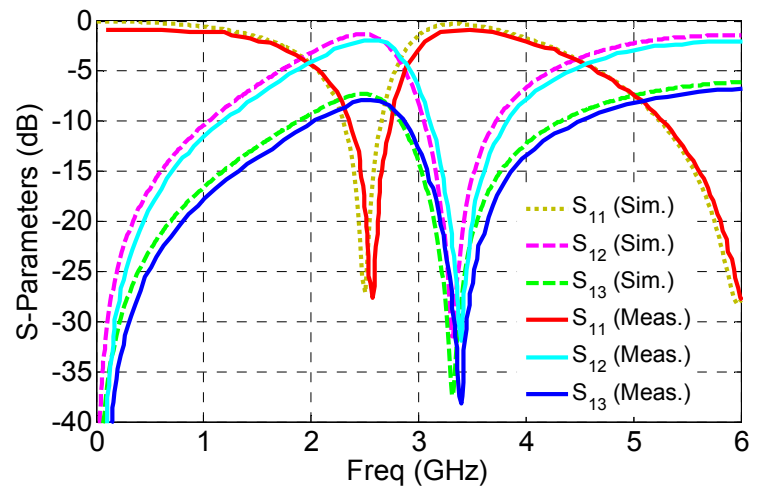

(b)

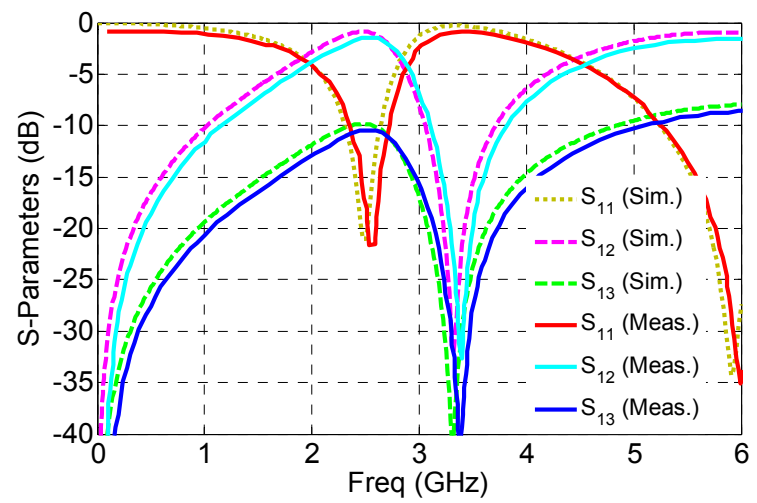

(c)

Fig. 14. Measured and simulated results of the fabricated equal/unequal HMSIW FPDs: (a) $\Delta$ out $=0 \mathrm{~dB}$, (b) $\Delta$ out $=6 \mathrm{~dB}$, (c) $\Delta$ out $=9 \mathrm{~dB}$.

The measured results well-matched with the simulated one. The equal FPD has a $3 \mathrm{~dB}$ bandwidth of $950 \mathrm{MHz}$ in $1.88-2.85 \mathrm{GHz}$ frequency band. The measured return loss is better than $28 \mathrm{~dB}$ and the measured in-band insertion losses $\mathrm{S}_{21}$ and $\mathrm{S}_{31}$ are $0.5 \mathrm{~dB}$. Moreover, the measured results demonstrate that the measured phase imbalances for the equal FPD are within $-5^{\circ}$ to $1.5^{\circ}$. The unequal power divider with $\Delta$ out $=6 \mathrm{~dB}$ has a $3 \mathrm{~dB}$ bandwidth of $960 \mathrm{MHz}$ in $1.86-2.82 \mathrm{GHz}$ frequency band. The measured in-band insertion loss and return loss are $0.4 \mathrm{~dB}$ and better than $27 \mathrm{~dB}$, respectively. In addition, the measured results illustrate that the measured phase imbalances for the unequal FPD with $\Delta$ out $=6 \mathrm{~dB}$ are within $2^{\circ}$ to $5.2^{\circ}$. The unequal power divider with $\Delta$ out $=9 \mathrm{~dB}$ has a $3 \mathrm{~dB}$ bandwidth of $920 \mathrm{MHz}$ in $1.88-2.80 \mathrm{GHz}$ frequency band. The measured in-band insertion loss and return loss are $0.5 \mathrm{~dB}$ and better than $22 \mathrm{~dB}$, respectively. Furthermore, the measured results prove that the measured phase imbalances for the unequal FPD with $\Delta$ out $=9 \mathrm{~dB}$ are within $-3.8^{\circ}$ to $-2.3^{\circ}$. The discrepancy between the measured and simulated results could be attributed to the tolerance of the dielectric constant, fabrication errors and the insertion loss of subminiature version A (SMA) connectors. Table 1 compares the performances of the proposed equal FPD with some recently reported FPDs. In this table, the advantages in size reduction and low insertion loss are very clear. Furthermore, the total size of the proposed FPDs are approximately $0.25,0.12$ and, 0.22 of the whole size of the reported FPDs in [7], [9] and [10], respectively.

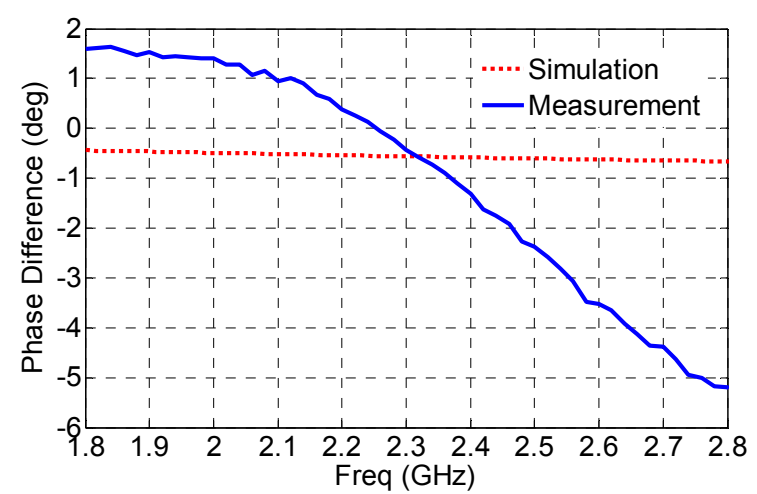

(a)

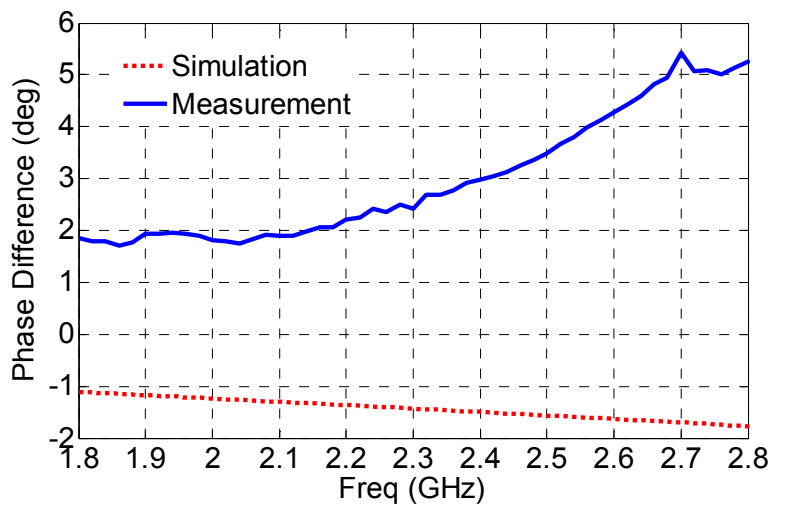

(b)

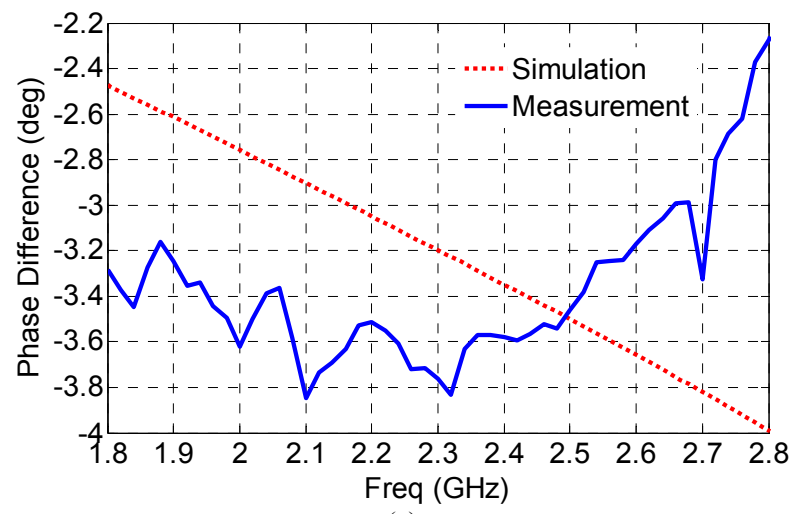

(c)

Fig. 15. Measured and simulated differential phases of the fabricated equal/unequal HMSIW FPDs: (a) $\Delta$ out $=0 \mathrm{~dB}$, (b) $\Delta$ out $=6 \mathrm{~dB}$, (c) $\Delta$ out $=9 \mathrm{~dB}$. 


\begin{tabular}{|c|c|c|c|c|c|}
\hline Ref. Num. & CF (GHz) & IL (dB) & RL (dB) & FBW $(\%)$ & Size $\left.\boldsymbol{(}_{\mathbf{g}}{ }^{2}\right)$ \\
\hline$[7]$ & 5.62 & 1 & 15 & 6 & 0.037 \\
\hline$[8]$ & 5.61 & 1.2 & 14 & 7.8 & 0.66 \\
\hline$[9]$ & 5.82 & 1.2 & 24 & 15.8 & 0.077 \\
\hline$[10]$ & 5.39 & 1.3 & 13 & 13.73 & 0.043 \\
\hline$[11]$ & 2.45 & 4.29 & 15 & 14.7 & 0.081 \\
\hline$[12]$ & 1.08 & 1 & 15 & 10.4 & 0.10 \\
\hline$[13]$ & 4.8 & 0.5 & 15 & 27 & 0.42 \\
\hline$[14]$ & 2.45 & 3.2 & 17 & 8.2 & 0.079 \\
\hline$[15]$ & 1.62 & 0.48 & 29 & 79.2 & 0.04 \\
\hline$[16]$ & 2.39 & 1.58 & 20 & 7.8 & 0.052 \\
\hline$[17]$ & 9 & 1.1 & 12 & 22.3 & 1.09 \\
\hline This work & 2.4 & 0.5 & 28 & 39.5 & 0.009 \\
\hline
\end{tabular}

Ref. Num.: Reference Number, CF: Center frequency, IL: Insertion loss, RL: Return loss, FBW: Fractional bandwidth.

Tab. 1. Comparison with other PDs.

\section{Conclusion}

In this paper, three equal/unequal PDs with filtering responses and arbitrary power dividing ratio based on the HMSIW structure and the miniaturized metamaterial unitcell have been introduced. To achieve filtering PDs with ultra-compact size, HMSIW configuration, evanescent mode propagation technique, metamaterial concept and stepped-impedance method have been used, simultaneously. By varying the situations of the output ports, the power division ratio can be easily tuned. Three HMSIW PDs with filtering response and different power division ratios of 1:1, 1:4, and 1:8 have been fabricated and tested. The measurement results approve the ability of the presented FPDs in term of size reduction. Moreover, the proposed FPDs have many benefits such as high selectivity, easy combination with other planar circuits, low cost and low loss.

\section{References}

[1] DANAEIAN, M., AFROOZ, K., HAKIMI, A. Miniaturization of substrate integrated waveguide filters using novel compact metamaterial unit-cells based on SIR technique. AEU-International Journal of Electronics and Communications, 2018, vol. 84, p. 62-73. DOI: 10.1016/j.aeue.2017.11.008

[2] DANAEIAN, M., AFROOZ, K. Compact metamaterial unit-cell based on stepped-impedance resonator technique and its application to miniaturize substrate integrated waveguide filter and diplexer. International Journal of $R F$ and Microwave Computer-Aided Engineering, 2019, vol. 29, no. 2, p. 1-13. DOI: 10.1002/mmce. 21537

[3] ZHU, Y., SONG, K., FAN, M., et al. Wideband single-ended-tobalanced power divider with intrinsic common-mode suppression. IEEE Microwave and Wireless Components Letters, 2020, vol. 30, no. 4, p. 379-382. DOI: 10.1109/LMWC.2020.2973863

[4] FENG, T., MA, K., WANG, Y. A self-packaged power divider with compact size and low loss. IEEE Transactions on Circuits and Systems II: Express Briefs, 2020, vol. 67, no. 11, p. 2437-2441. DOI: 10.1109/TCSII.2020.2965970

[5] HAYATI, M., ZARGHAMI, S. Analysis of asymmetric coupling lines and design of a Wilkinson power divider based on harmonic suppression network. AEU-International Journal of Electronics and Communications, 2020, vol. 115, p. 1-11. DOI: 10.1016/j.aeue.2019.153047

[6] MOZNEBI, A.-R., AFroOZ, K., DANAEIAN, M., et al. Fourway filtering power divider using SIW and eighth-mode SIW cavities with ultra-wide out-of-band rejection. IEEE Microwave and Wireless Components Letters, 2019, vol. 29, no. 9, p. 586-588. DOI: 10.1109/LMWC.2019.2931115

[7] MOZNEBI, A.-R., DANAEIAN, M., ZAREZADEH, E., et al. Ultra-compact two-way and four-way SIW/HMSIW power dividers loaded by complementary split-ring resonators. International Journal of $R F$ and Microwave Computer-Aided Engineering, 2019, vol. 29, no. 10, p. 1-8. DOI: $10.1002 /$ mmce. 21878

[8] MOZNEBI, A.-R., AFROOZ, K., DANAEIAN, M., et al. Compact filtering power divider based on corrugated third-mode circular SIW cavities. Microwave and Optical Technology Letters, 2020, vol. 62 , no. 5, p. 1900-1905. DOI: 10.1002/mop.32259

[9] DANAEIAN, M., MOZNEBI, A.-R., AFROOZ, K., et al. Miniaturized equal/unequal SIW power divider with bandpass response loaded by CSRRs. Electronics Letters, 2016, vol. 52, no. 22, p. 1864-1866. DOI: 10.1049/el.2016.2203

[10] DANAEIAN, M., MOZNEBI, A.-R., AFROOZ, K., et al. Miniaturized filtering SIW power divider with arbitrary powerdividing ratio loaded by open complementary split-ring resonators. International Journal of Microwave and Wireless Technologies, 2017, vol. 9, no. 9, p. 1827-1832. DOI: $10.1017 / \mathrm{S} 175907871700071 \mathrm{X}$

[11] CAO, Q., LIU, H., GAO, L. Design of high selectivity filtering power divider with high out-of-band rejection. Electromagnetics, 2019, vol. 39, no. 7, p. 473-480. DOI: $10.1080 / 02726343.2019 .1658162$

[12] LU, D., YU, M., BARKER, N. S., et al. A simple and general method for filtering power divider with frequency-fixed and frequency-tunable fully canonical filtering-response demonstrations. IEEE Transactions on Microwave Theory and Techniques, 2019, vol. 67, no. 5, p. 1812-1825. DOI: 10.1109/TMTT.2019.2903504

[13] WANG, X., ZHU, X-W., TIAN, L., et al. Design and experiment of filtering power divider based on shielded HMSIW/QMSIW technology for 5G wireless applications. IEEE Access, 2019, vol. 7, p. 72411-72419. DOI: 10.1109/ACCESS.2019.2920150

[14] SHERAFAT VAVIRI, H., ZARGHAMI, S., SHAMA, F., et al. Compact bandpass Wilkinson power divider with harmonics suppression. AEU-International Journal of Electronics and Communications, 2020, vol. 117, p. 1-8. DOI: 10.1016/j.aeue.2020.153107

[15] DONG, J., SHI, J., XU, K. Compact wideband differential filtering power divider based on three-line coupled structure with lumped 
elements. Electronics Letters, 2020, vol. 56, no. 12, p. 609-611. DOI: $10.1049 / \mathrm{el} .2020 .0475$

[16] LUO, M., TANG, X. H., XU, X., et al. Filtering power divider with good output balance and unsymmetrical structure. Microwave and Optical Technology Letters, 2020, vol. 62, no. 4, p. $1557-1563$. DOI: $10.1002 /$ mop. 32237

[17] HE, Z., YOU, C. J., LENG, S., et al. Compact power divider with improved isolation and bandpass response. Microwave and Optical Technology Letters, 2017, vol. 59, no. 7, p. 1776-1781. DOI: 10.1002/mop.30621

[18] DURAISAMY, T., BARIK, R. K., SHOLAMPETTAI SUBRAMANIAN, K., et al. A novel SIW based dual-band power divider using double-circular complementary split ring resonators. Microwave and Optical Technology Letters, 2019, vol. 61, no. 6, p. 1529-1533. DOI: 10.1002/mop.31772

[19] BARIK, R. K., CHENG, Q. S., PRADHAN, N. C., et al. A compact SIW power divider for dual-band applications. Radioengineering, 2020, vol.29, no. 1, p. 94-100. DOI: $10.13164 / \mathrm{re} .2020 .0094$

[20] LIU, B., LYU, Y. P., ZHU, L., et al. A compact triple-mode bandpass filter with wide-stopband using half-mode substrate integrated waveguide cavity loaded with slots. Microwave and Optical Technology Letters, 2020, vol. 62, no. 3, p. 1056-1059. DOI: $10.1002 /$ mop. 32124

[21] SUN, Q., BAN, Y. L., LIAN, J. W., et al. Millimeter-wave multi beam antenna based on folded C-type SIW. IEEE Transactions on Antennas and Propagation, 2020, vol. 68, no. 5, p. 3465-3476. DOI: 10.1109/TAP.2020.2966050
[22] HUANG, L., CHA, H., ZHANG, S. Compact wideband-folded ridge substrate-integrated waveguide filter. IEEE Microwave and Wireless Components Letters, 2020, vol. 30, no. 3, p. 241-244. DOI: 10.1109/LMWC.2020.2971659

[23] DANAEIAN, M., MOZNEBI, A.-R., AFROOZ, K. A novel super compact half-mode substrate integrated waveguide filter using modified complementary split-ring resonator. International Journal of RF and Microwave Computer-Aided Engineering, 2019, vol. 29, no. 6, p. 1-8. DOI: 10.1002/mmce.21709

[24] POZAR, D. M. Microwave Engineering. $4^{\text {th }}$ ed. University of Massachusetts at Amherst: John Wiley \& Sons, 2012. ISBN-13: 978-0470631553

\section{About the Authors ...}

Mostafa DANAEIAN was born in Yazd, Iran, in 1985. He received the B.Sc. degree in Electrical Engineering from Yazd University, Yazd, Iran, in 2008, the M.Sc. and Ph.D. degrees from the Shahid Bahonar University of Kerman, Kerman, Iran, in 2011 and 2016, all in Electrical Engineering. He is currently an Assistant Professor with the Electrical Engineering Department, Vali-e-Asr University of Rafsanjan, Rafsanjan, Iran. His research interests are metamaterial transmission lines, $\mathrm{RF} /$ microwave circuits design. He is currently involved with microwave filters, power dividers and, diplexers based on CRLH structures. 